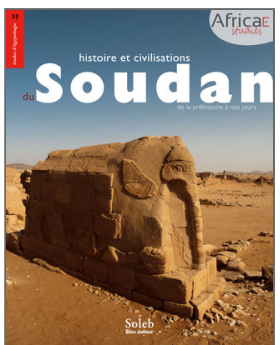

Olivier Cabon (dir.)

Histoire et civilisation du Soudan

De la préhistoire à nos jours

\title{
1. Nil Blanc, Nil Bleu, Nil Jaune
}

Le Soudan à l'aube de l'histoire

\section{Claude Rilly}

DOI : 10.4000/books.africae.2787

Éditeur: Africae, Soleb, Bleu autour

Lieu d'édition : Paris, Khartoum

Année d'édition : 2017

Date de mise en ligne : 17 janvier 2022

Collection : Africae Studies

EAN électronique : 9782493207074

\section{OpenEdition}

\author{
Books
}

http://books.openedition.org

\section{Référence électronique}

RILLY, Claude. 1. Nil Blanc, Nil Bleu, Nil Jaune : Le Soudan à l'aube de l'histoire In : Histoire et civilisation du Soudan : De la préhistoire à nos jours [en ligne]. Paris, Khartoum : Africae, 2017 (généré le 28 janvier 2022). Disponible sur Internet : <http://books.openedition.org/africae/2787>. ISBN : 9782493207074. DOI : https://doi.org/10.4000/books.africae.2787. 


\section{Nil Blanc, Nil Bleu, Nil Jaune}

le Soudan à l'aube de I'histoire 
Bien qu'il soit entouré de pays où se concentrent les témoins fossiles des premiers âges de l'humanité — Éthiopie, Kenya et Tchad —, le Soudan est relativement pauvre en traces très anciennes de présence humaine. Par exemple, le crâne humain découvert à Singa, sur le Nil Bleu, daté d'environ 150000 ans, appartenait déjà à une forme archaïque d'Homo sapiens. Des témoignages plus anciens, mais découverts hors contexte archéologique, sont des galets grossièrement taillés retrouvés en Nubie par l'équipe suisse de Kerma et qui typologiquement peuvent remonter vers 800000 ans. Il fait pourtant peu de doute que le territoire soudanais ait abrité des populations humaines parmi les premières du globe, eu égard à la proximité de la vallée du Rift où l'on a découvert jusqu'à présent la plupart des formes d'hominidés les plus anciennes.

Cette pauvreté s'explique par plusieurs facteurs. Tout d'abord, les terrains volcaniques, les plus propices à piéger les fossiles, sont fréquents en Éthiopie et au Kenya mais rares au Soudan. D’autre part, le cours du Nil a souvent varié et nombre de sites paléolithiques anciens ont été ennoyés, tandis que d'autres ont été recouverts par des millénaires de dépôts alluvionnaires. Enfin, les archéologues se sont longtemps focalisés sur le très riche patrimoine historique, si bien qu'assez peu de recherches ont été effectuées sur les périodes précédentes jusqu'aux fouilles de sauvetage de la Nubie organisées par l'Unesco dans les années 1960.

\section{Le Paléolithique et le Mésolithique soudanais}

Une exception doit être faite pour Anthony J. Arkell, le premier commissioner for archaeology du Soudan sous domination anglo-égyptienne. Au début $\mathrm{du} \mathrm{xx}^{\mathrm{e}}$ siècle, il déploya une énergie infatigable pour parcourir le pays à la recherche des vestiges de son passé. C'est néanmoins à proximité de Khartoum qu'il fut le premier à découvrir et étudier des sites préhistoriques. Près d'Omdurman, il explora le site de Khor Abou Anga, dont les vestiges s'étageaient du Paléolithique moyen au Paléolithique supérieur (120000 à 40000 ans). L'artefact emblématique de ces premières cultures est le biface, outil à tout faire des époques anciennes, depuis le fouissage du sol jusqu'au taillage du bois et de l'os.

Les techniques utilisées pour la taille étaient typiques de la période acheuléenne. Ce premier site montrait donc que, si le Soudan préhistorique présentait déjà des particularités, il participait des grandes cultures humaines répandues sur l'ensemble de l'ancien monde et notamment du reste de l'Afrique. Après Arkell, de nombreux sites acheuléens et datant du Paléolithique moyen furent découverts près de Wadi Halfa, à la frontière avec l'Égypte, lors des fouilles de sauvetage que nécessita 
l'érection du barrage d'Assouan et à Khasm el-Girba, un des lieux sur le Haut-Atbara (non loin de la frontière avec l'Érythrée) où furent relogés les Nubiens chassés de leurs terres par le lac de retenue du barrage.

L'île de Saï, au nord du Soudan (voir p. 524 sq.), présente un site paléolithique exceptionnel qui fut exploité plus tard, bien que déjà repéré par Arkell. De fines alluvions déposées régulièrement ont ici piégé un établissement situé au pied du Gébel Adou, le point culminant de l'île, et malgré les fréquents remodelages qu'elle a connus, les vestiges ont été préservés. À la fin des années 1990, une équipe de l'université de Louvain sous la direction de Philip van Peer y a mis en évidence, au prix de très profondes excavations, un habitat remontant à plus de 200000 ans, d'abord occupé lors de périodes humides par des hommes de l'horizon acheuléen et remplacés vers 150000 , une phase plus sèche, par une culture connue sous le nom de «sangoen", présente de l'Afrique du Sud à l'Éthiopie. Dans les strates anciennes, des meules en grès, témoignant de la transformation de graminées sauvages très rarement attestée au Paléolithique ancien, voisinent avec des haches de grès ou de quartz. Par la suite, l'outillage de pierre sangoen évolue vers des formes plus légères et tranchantes, utilisées sans doute comme armes pour la chasse qui, avec l'aridité croissante, était devenue un moyen de subsistance indispensable.

Mais la découverte la plus remarquable effectuée sur le site paléolithique de Saï est celle de nodules d'ocre jaune et rouge et des galets qui ont servi à les broyer. C'est une des toutes premières attestations de l'utilisation de pigments par les hommes préhistoriques. On ignore en revanche leur usage. L'apparition des peintures rupestres ne se fera en effet que beaucoup plus tard. Peut-être ces pigments étaient-ils employés pour des décorations corporelles, qui ont été en usage au Soudan jusqu'à l'époque moderne, notamment dans les monts Nouba.

La période suivante du Paléolithique inférieur voit le Nil, au terme de variations multiples, se stabiliser dans son cours actuel. Selon certains chercheurs, le Nil Bleu n'aurait rejoint le bassin du fleuve principal que vers 70000 avant notre ère. Les vestiges de l'occupation humaine sont attestés uniquement en Basse-Nubie, avec des horizons locaux. Vers 36000 , le Khormusien (d'après le site de Khor Musa, au sud de Wadi Halfa) est caractérisé par une industrie lithique particulièrement fine, qui gagne encore en précision durant l'Halfien (de Wadi Halfa), daté de 19000 avant notre ère. Sur la rive gauche de Wadi Halfa, un horizon plus récent (15000 à 8000 ), le Qadien (d'après le site de Qada), a livré des témoignages d'innovations technologiques qui préfigurent les périodes suivantes: utilisation de microlithes, généralisation de l'usage de meules et apparition de la poterie. C'est également de cette époque que datent les premiers cimetières connus en Nubie, notamment au Gébel 


\section{de la préhistoire au royaume de Kerma}

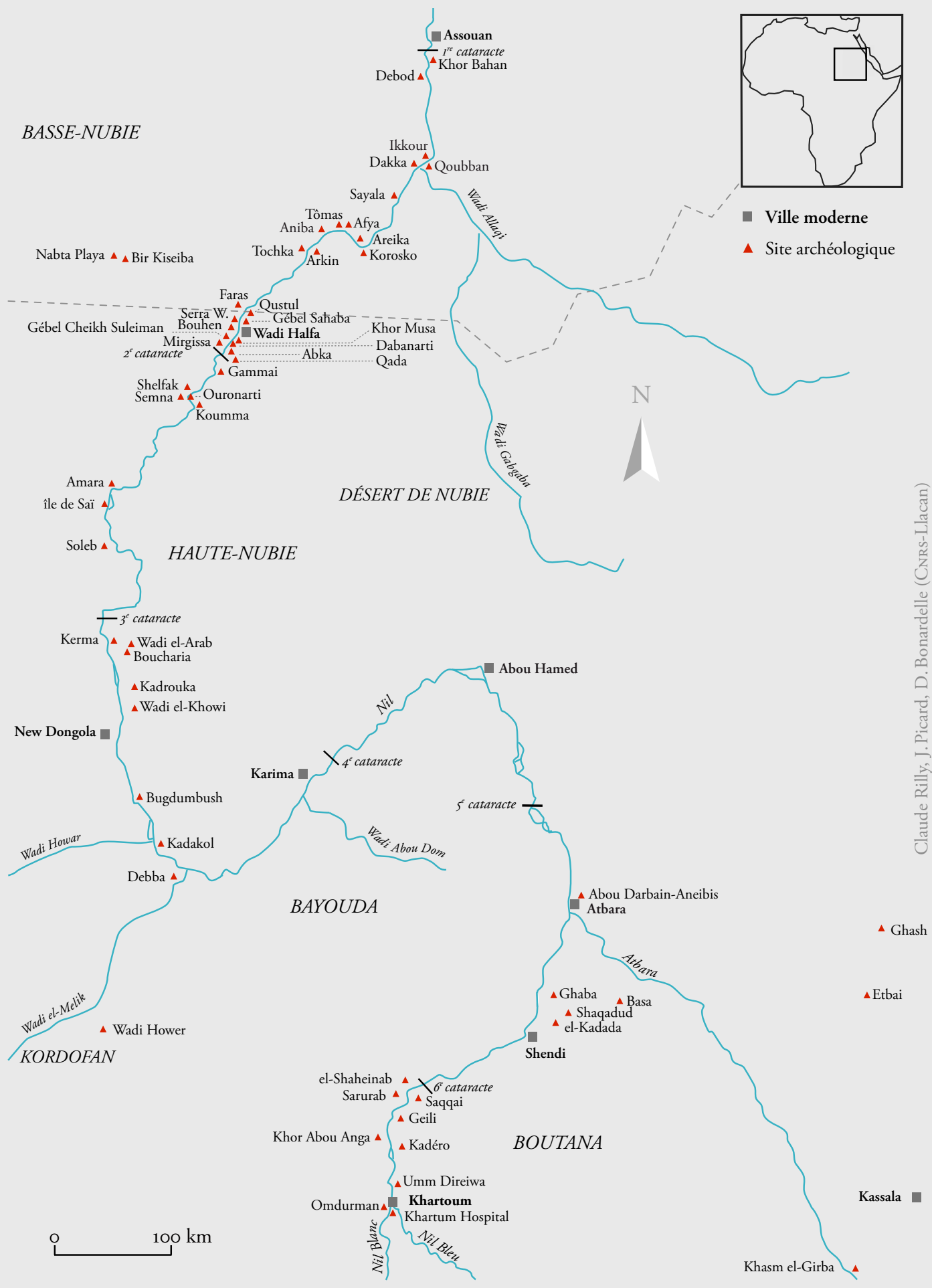



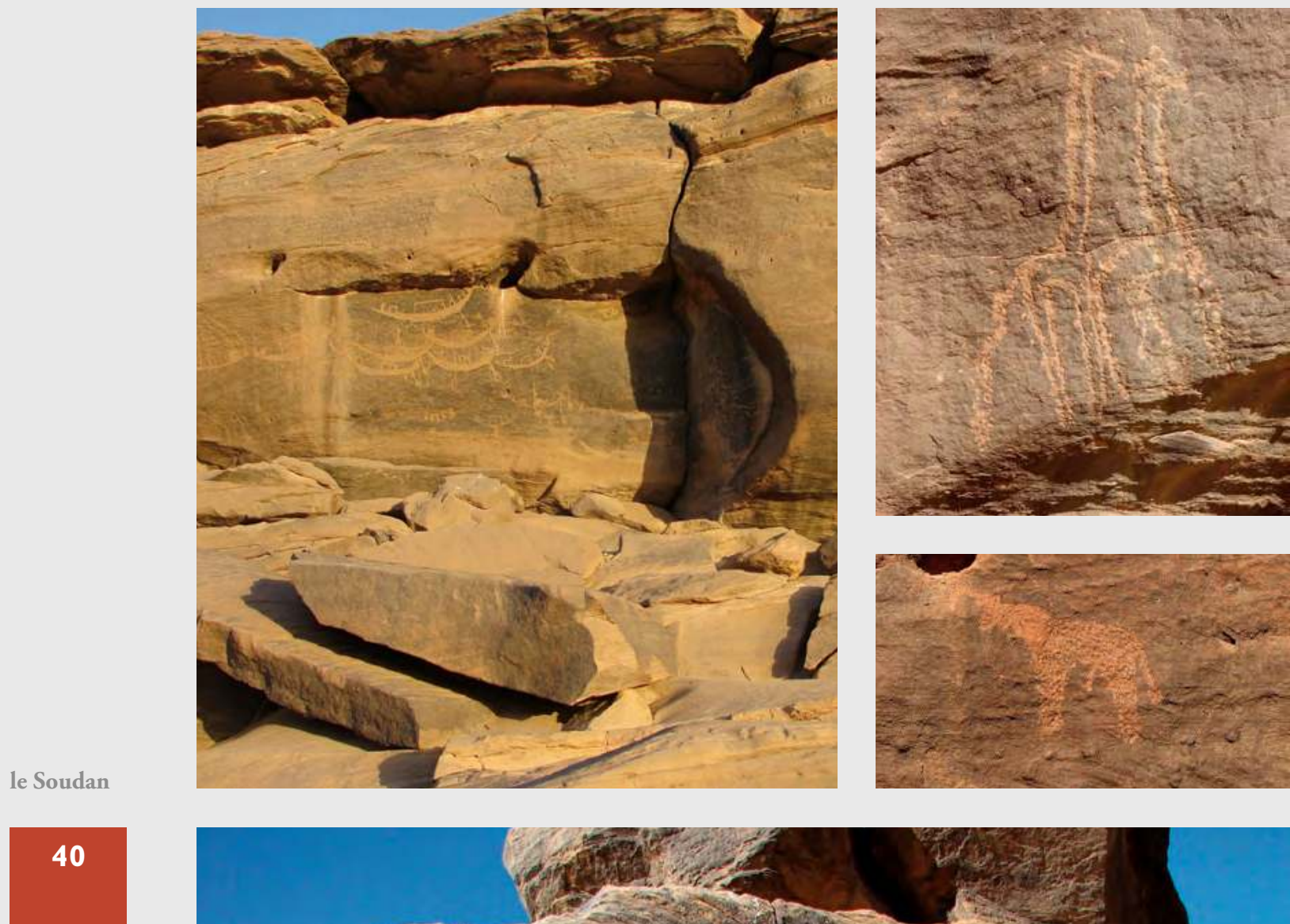

des origines à la chute du sultanat Fung

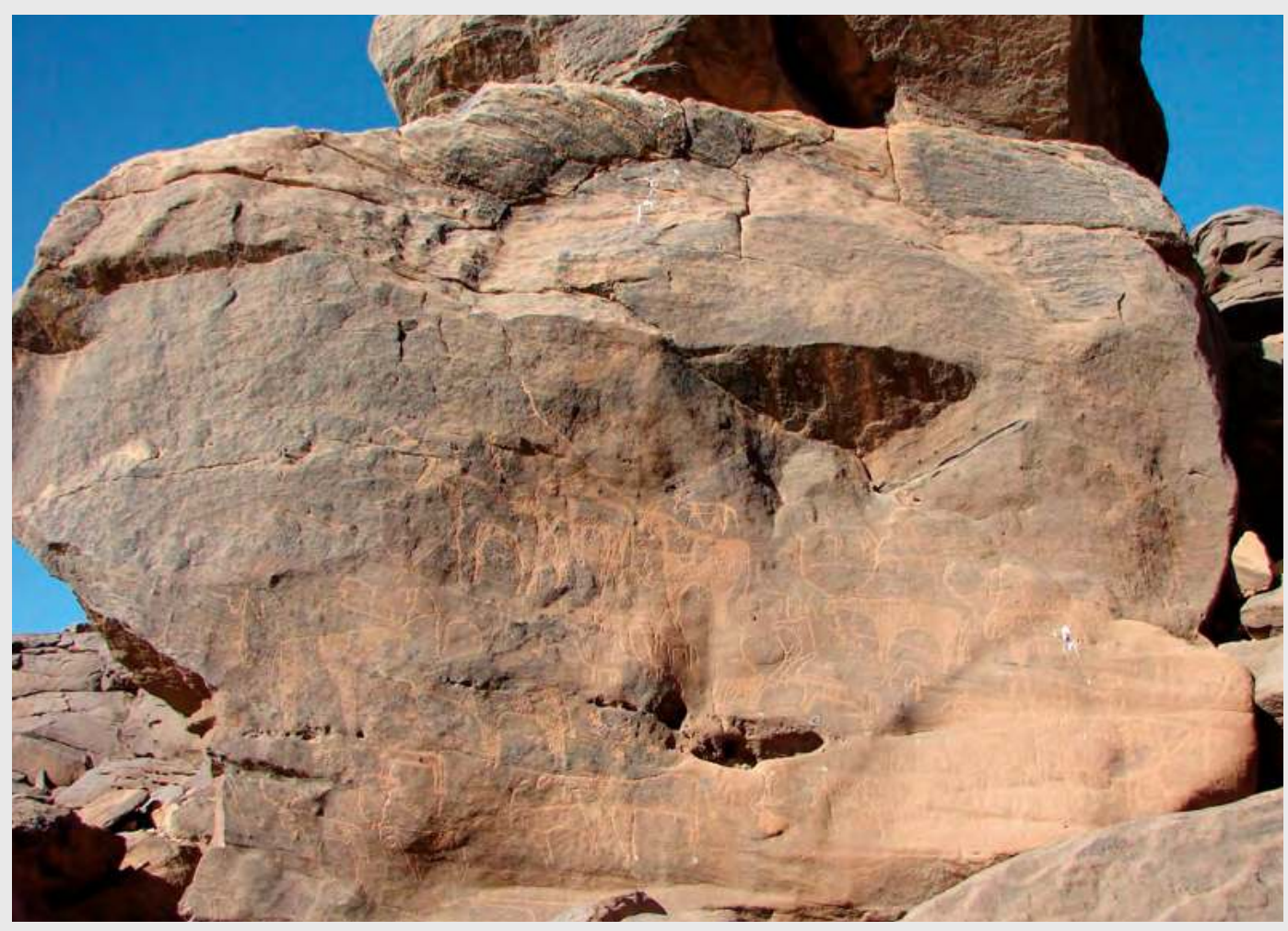


Sahaba (nord de Wadi Halfa, vers 12000-10000 av.J.-C.), où 55 squelettes ont été découverts inhumés dans des fosses recouvertes de dalles de pierre. La présence d'éclats taillés au milieu des restes humains, parfois retrouvés enfoncés dans l'os, suggère que certains de ces individus ont été victimes de violences, peut-être causées par des conflits entre tribus.

La transition entre Paléolithique et Néolithique, le Mésolithique (8000-5000 avant notre ère), est une période cruciale dans le développement des sociétés humaines dans la vallée du Nil. Sur le site où se dresse aujourd'hui l'hôpital de Khartoum, Arkell repéra des artefacts anciens dans les années 1940 et y entreprit des fouilles qui lui permirent d'identifier un horizon appelé "Mésolithique de Khartoum». La principale caractéristique de cette culture, commune à toute la vallée du Nil moyen, est de superposer à une économie de chasseurs-cueilleurs l'utilisation de la céramique, que l'on pensait alors liée intrinsèquement à une société d'agriculteurs. Ces premières poteries élaborées sont décorées de lignes ondulées (Wavy Line), plus tard réalisées en pointillés (Dotted Wavy Line). Outre la cueillette et la chasse, la pêche joue un rôle important et les sites abondent en harpons taillés dans l'os. Le Soudan, en effet, connaît alors, après une période d'aridité extrême vers 10000 avant notre ère, un long épisode pluvieux connu sous le nom de «Grand Humide» qui va durer jusque vers le sixième millénaire et pendant lequel, là où aujourd'hui ne s'étend que le désert, rivières et lacs abondent, regorgeant de poissons et de mollusques. Au nord du Soudan, le Mésolithique de Khartoum présente un faciès local appelé «Khartoum Variant». Il s'agit là aussi de populations semi-nomades, organisées en petits campements saisonniers, généralement situés assez loin du fleuve et de ses crues alors dévastatrices. Les défunts sont inhumés tout près des zones habitées.

Dans ce cadre général du Mésolithique de Nubie, les récentes fouilles

Wadi Sabo:

bateaux prédynastiques, girafes, éléphant et bovidés. de la mission suisse de Matthieu Honegger ont mis en évidence plusieurs sites autour de Kerma. Sur le plus ancien, Boucharia, daté de 8300 av. J.-C., ont été trouvés les premiers vestiges de céramique actuellement connus en Nubie. Leurs décors en lignes droites pointillées ne sont pas attestés ailleurs mais préfigurent peut-être la Dotted Wavy Line qui apparait dans la région au millénaire suivant. Situé à l'est de Kerma, dans une zone de gébels aujourd'hui désertique, Wadi el-Arab était le lieu d'un campement formé de cabanes, dont la principale était renforcée par une profonde fondation creusée dans le roc, ce qui indique un premier pas vers la sédentarisation. Ici aussi, les défunts étaient placés dans des fosses sous l'habitat ou en bordure immédiate. Les datations sur ce site sans cesse réutilisé s'étalent de 8000 à 6000 av. J.-C. Mais l'élément le plus étonnant retrouvé par les fouilleurs est la présence de crânes de bovins qui semblent bien être des animaux domestiques. Ce point a confirmé une première découverte faite peu auparavant dans les tombes du site d'el-Barga 
(près de Kerma), où des restes similaires ont été datés de 6800 av. J.-C. Il s'agit des premières attestations de domestication animale sur le sol africain et seuls quelques rares sites comme l'Acacus en Libye ou Nabta Playa et Bir Kiseiba dans le désert occidental au sud de l'Égypte présentent des traces aussi anciennes de bovins domestiqués. Certains préhistoriens proposent donc que, contrairement aux ovins et aux caprins, introduits en Afrique depuis le ProcheOrient au sixième millénaire, la domestication du bœuf s'est faite sur place, à partir d'une souche locale d'aurochs (Bos Primigenius).

\section{Le Néolithique soudanais}

le Soudan

\section{2}

des origines

à la chute

du sultanat

Fung

Les principales innovations qui en Europe accompagnent l'émergence du Néolithique, c'est-à-dire l'introduction de l'élevage, l'apparition de la céramique, la constitution de villages, étaient donc déjà en place au Soudan dès le septième millénaire. La technique de la pierre polie est de plus déjà attestée dans la joaillerie (notamment des ornements de lèvre en amazonite et en cornaline) retrouvée dans les tombes mésolithiques d'el-Barga (vers 6000 av. J.-C.). L'agriculture stricto sensu, caractérisée par la sélection, la domestication et la culture d'espèces végétales, n’apparaîtra que bien plus tard, au quatrième millénaire, comme l'attestent les greniers Pré-Kerma de l'île de Saï. Il faudrait en toute logique faire remonter au septième millénaire le début du Néolithique et s'abstraire des calques européens qui y incluent l'agriculture. Ici encore, la tradition des préhistoriens suit les classifications proposées par Arkell, qui faisait commencer le Néolithique par la culture qu'il découvrit en 1949 sur le site d'el-Shaheinab, au nord de Khartoum, datée d'environ 4900 à 3800 av. J.-C. Mais on sait désormais que la culture d'el-Shaheinab n'est qu'une phase récente du Néolithique soudanais.

Cette période a au moins en commun avec le Néolithique procheoriental et européen l'accroissement de la population, lié à l'augmentation des moyens de subsistance et notamment du cheptel. Si l'on calcule le pourcentage des ossements animaux retrouvés sur les sites appartenant à des espèces domestiquées, on passe de $20 \%$ pour le Néolithique ancien à $80 \%$ pour le Néolithique récent. Les bovins ne sont d'ailleurs plus les seules espèces présentes, puisque les ovins et les caprins, moins exigeants en eau et en nourriture, ont été introduits depuis le Levant. De vastes cimetières apparaissent à el-Shaheinab, Kadéro (banlieue nord de Khartoum), Ghaba et el-Kadada (près de Shendi), Kadrouka (Moyenne-Nubie, au sud de Kerma). Groupant plusieurs dizaines à plusieurs centaines de tombes, ils ont livré un matériel de qualité extraordinaire, souvent réalisé spécifiquement pour le contexte funéraire. Les plus richement dotées 


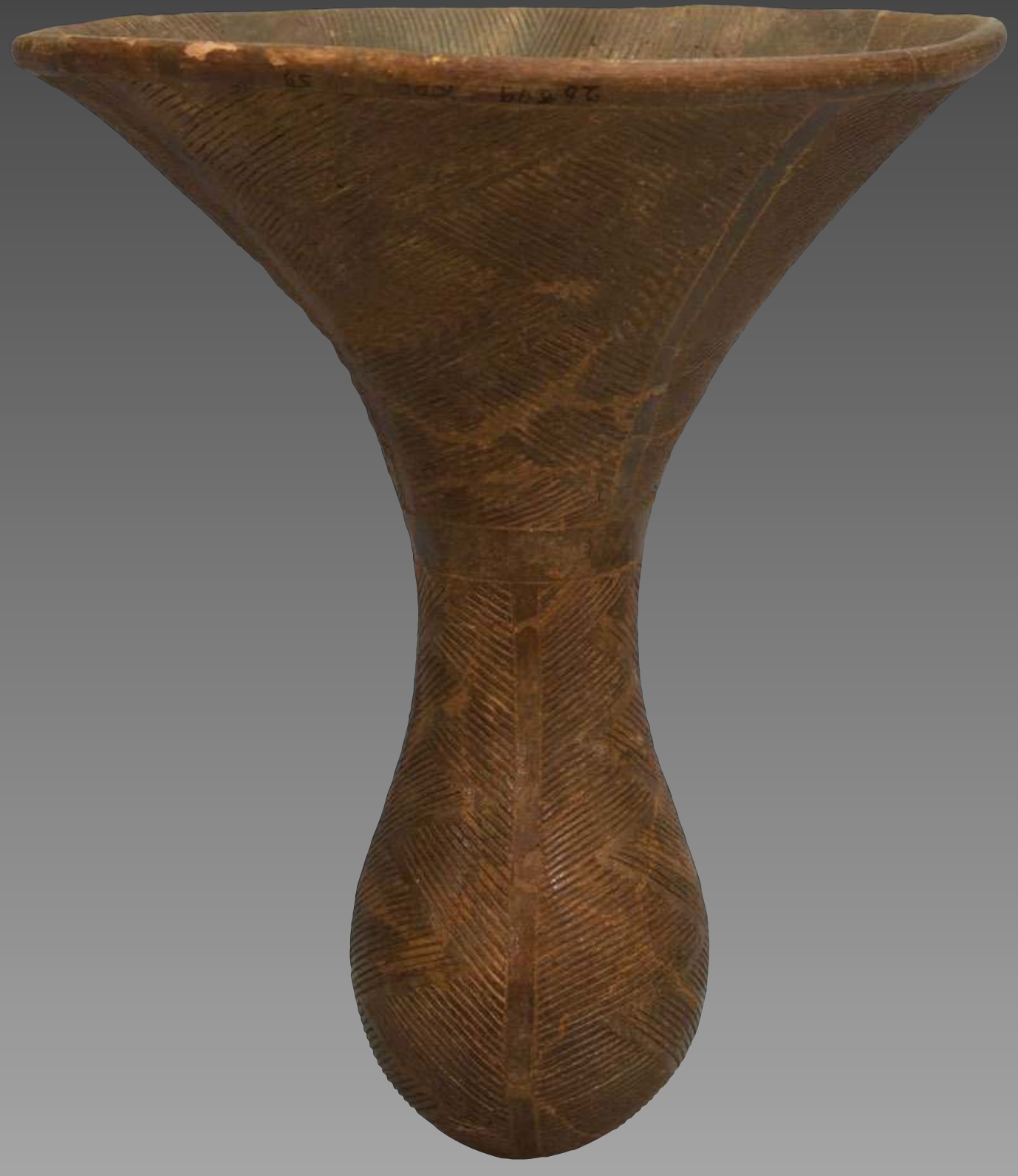

Vase « caliciforme », el-Kadada, cimetière C, tombe 3, KDD 76/3/59, Néolithique,

3610-3392 av. J.-C. (calibrated), musée national, Khartoum, SNM 26899. Voir également p. 465. 


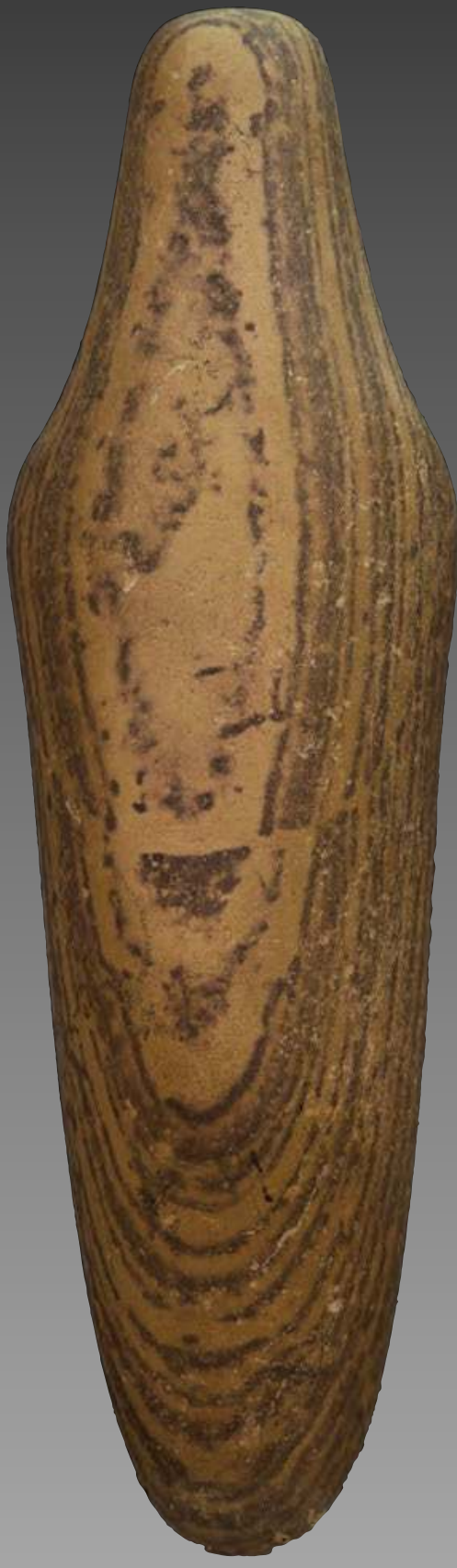

Figurine féminine stylisée, Kadrouka, cimetière 1, tombe 131, KDK 1/131/8, musée national, Khartoum, SNM 26861. Voir également p. 465. 
parmi ces sépultures sont le plus souvent associées à un individu, homme ou femme, qui disposait manifestement d'un statut supérieur, montrant une hiérarchisation de la société dont on n'a pas de trace auparavant. Elles occupent d'ailleurs une place privilégiée au centre des inhumations. Ainsi la tombe 131 du cimetière 1 de Kadrouka, fouillée par le préhistorien français Jacques Reinold et datée par le radiocarbone de 4200 av. J.-C., a-t-elle livré, autour du squelette d'un homme d'une quarantaine d'années, un vase orné dit «caliciforme», typique des tombes d'élite, une grande jarre, neuf têtes de massues en pierre polie, deux peignes et un étui à fard en ivoire d'hippopotame, plusieurs bracelets taillés dans des défenses d'éléphant, deux bucranes (crânes de bovins) enduits de pigment blanc et une figurine féminine stylisée. Cette statuette, taillée dans un grès lité dont les veines ont été habilement utilisées pour mettre en valeur les reliefs, et aux traits $\mathrm{du}$ visage simplement esquissés par des incisions dans la pierre, compte parmi les chefs-d'œuvre mondiaux de l'art néolithique. Cette hiérarchisation ne concernait pas seulement des individus, mais aussi leur famille, comme le montre le trousseau funéraire luxueux d'un enfant de trois ans retrouvé dans le même cimetière, qui avait été inhumé avec quatre vases, un bucrane, un bracelet, un collier, trois labrets, un étui à fard en ivoire et une hache polie. Il est rarissime, toutes époques confondues, qu'un enfant en bas âge ait droit à un tel équipement funéraire.

De ces sociétés néolithiques, on ne connaît que peu d'éléments en dehors de la culture matérielle révélée par les tombes. L'habitat a été peu fouillé car les traces légères qu'il laisse (trous de poteaux comblés de sédiments, fonds de cabane, tessons) sont difficiles à repérer. On sait que l'on a affaire à des populations partiellement sédentarisées, qui toutefois possèdent des campements secondaires saisonniers pour la pâture du bétail et pour la pêche, pratiquée au moment des basses eaux. Une certaine spécialisation des tâches se fait jour vers la fin du Néolithique: la tombe d'un jeune adulte à Kadéro contenait par exemple un outillage complet pour le débitage des pierres taillées. De leur religion, on sait peu de choses. Les bovins, dont les crânes accompagnent le défunt, semblent avoir joué un rôle important dans les rituels, préfigurant en cela les sociétés protohistoriques et le royaume de Kerma. La nécropole d'el-Kadada, près de Shendi, a récemment permis de mettre en évidence les premiers morts d'accompagnement attestés au Soudan. Le terme "sacrifice humain" parfois utilisé est abusif, car il s'agit non pas de personnes offertes par un tiers au défunt comme des animaux sacrifiés, mais de membres de sa maisonnée qui l'accompagnent dans la mort au même titre que son trousseau funéraire. Cette pratique sera récurrente dans les civilisations successives du Nil moyen (notamment Kerma et postméroïtique). 
La description du Néolithique soudanais ne saurait se réduire à la vallée du Nil. Vers 8500 av. J.-C., le Grand Humide, longue période d'importante pluviosité, se mit en place en quelques générations sur le Sahara. Ce changement dû aux modifications du trajet des pluies tropicales d'été fit remonter les savanes herbeuses jusqu'au sud de l'Égypte, créant un «Sahara vert». De nombreux lacs s'y formèrent et les oueds devinrent de véritables rivières. Ce nouvel environnement attira des populations de chasseurs-cueilleurs, peut-être aussi repoussées de zones méridionales devenues trop humides et malsaines ou des rives d'un Nil désormais énorme et dangereux. Vers 5300 av. J.-C., la tendance s'inverse et l'on s'achemine peu à peu vers l'aridité qui caractérise le Sahara aujourd'hui. Les populations qui habitaient le désert Libyque au sud de l'Égypte et qui avaient adopté l'élevage des bovins se dispersèrent peu à peu vers l'ouest (le Gilf Kebir, célèbre pour ses abris ornés de peintures rupestres), vers l'est (le Nil) ou vers le sud. C'est justement au sud que se trouvait un grand affluent du Nil, le Wadi Howar, aujourd'hui presque entièrement disparu sous les sables, au point que l'explorateur Leo Frobenius l'avait qualifié de "Nil Jaune». De nos jours, seul le haut Wadi Howar, dans le Darfour, connaît encore un remplissage saisonnier. Il prend sa source au sud du massif de l'Ennedi, au Tchad. De l'autre côté, après un parcours de près de $1200 \mathrm{~km}$, il se jetait dans le Nil à el-Debba, à $100 \mathrm{~km}$ au sud de Kerma. Les recherches dans la région du Wadi Howar effectuées par l'université de Cologne (projet Acacia) ont montré que lorsque la désertification du Sahara oriental s'est produite, entre 5000 et 3500 av. J.-C., ce tributaire du Nil est longtemps resté en eau, attirant des populations nombreuses particulièrement depuis le nord, avant que son cours ne se disjoigne puis finisse par devenir temporaire vers le milieu du deuxième millénaire. Le Wadi Howar, durant les trois millénaires où il fut occupé, fut densément peuplé, comme en témoignent les quelque 1700 sites archéologiques qui y ont été repérés par l'équipe de Cologne. Il faut ajouter au bassin de la rivière quelques zones proches comme le Gébel Tageru au sud, l'erg Ennedi au nord et le massif de l'Ennedi à l'ouest.

Trois phases d'occupation ont été définies. De 5000 à 4000 av. J.-C. (phase 1), l'ensemble de la rivière est en eau et ses berges accueillent des campements de chasseurs-cueilleurs, qui consomment également poissons et mollusques. De 4000 à 2200 av. J.-C. (phase 2), le cours inférieur du wadi, vers le Nil, s'assèche. Les populations, dont une bonne partie provient certainement des régions environnantes gagnées par la désertification, sont désormais des éleveurs de bovins, les ovins et les caprins n'étant introduits que vers la fin de cette période. Des contacts avec la vallée du Nil sont attestés par la présence sur les sites de céramique de type "herringbone» (décors en "arêtes de poissons»). De 2200 
à 1100 av. J.-C., c'est l'ensemble du wadi qui est gagné par l'assèchement, saisonnier dans le haut bassin et quelques endroits du cours moyen, quasi permanent ailleurs. Les sites, encore nombreux, sont plus dispersés. Les caprins et les ovins, plus sobres que les bovins, jouent désormais un rôle prépondérant. L'âne, connu au Soudan depuis au moins 2500 av. J.-C., permet des déplacements plus faciles et favorise la nomadisation. Après 1100 av. J.-C., la région cesse définitivement d'être habitable, à l'exception du haut bassin situé sous l'Ennedi.

Le Wadi Howar a probablement joué un rôle essentiel dans l'histoire du Soudan néolithique et protohistorique. Il constituait alors un moyen de communication facile entre le Nil et le bassin du Tchad. Après sa dessiccation, son lit ensablé restera d'ailleurs une piste est-ouest très pratiquée. C'était d'autre part un réservoir de population probablement à l'origine de la migration vers les rives du Nil moyen de deux groupes ethniques apparentés, les Koushites au troisième millénaire et, au début de notre ère, au terme de longues pérégrinations à travers le Darfour et le Kordofan, les Nubiens

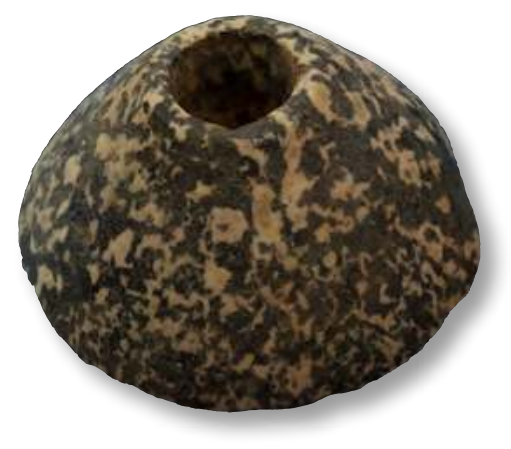

Tête de massue,

musée national, Khartoum.

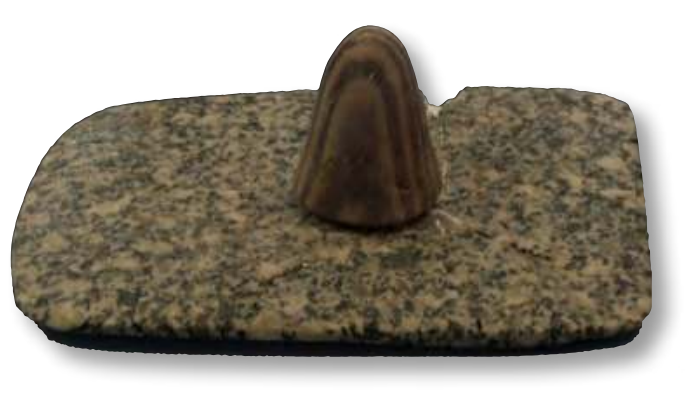

Palette,

musée national, Khartoum. 